\title{
A Bridge Between Cultures: A RePORT ON THE PROCESS OF TRANSLATING THE EQ-5D INSTRUMENT INTO SHONA
}

\begin{abstract}
There is an increasing need in medical research for outcome measures that are both locally applicable and internationally recognised. The European Quality of Life 5-dimensions (EQ-5D) has been found to be a valid and reliable instrument for describing health related quality of life in Western societies. The paper describes the process of translating the EQ-5D into Shona, the language spoken by the majority of Zimbabweans. The EuroQoL group has developed a protocol for translation that was followed rigorously. Issues such as conceptualisation of health concepts cross-culturally, semantic equivalence (the transfer of meaning across languages) and specific idiomatic expressions are

\section{JELSMA J'; CHIVAURA V';} DE WEERDT $W^{3}$; DE COCK $P^{4}$;

1 Department of Rehabilitation, University of Zimbabwe. Faculteit Lichamelijke Opvoeding en Kinesitherapie, Katholieke Universiteit Leuven, Belgium.

2 Department of English, University of Zimbabwe.

${ }^{3}$ Faculteit Lichamelijke Opvoeding en Kinesitherapie, Katholieke Universiteit Leuven, Belgium.

${ }^{4}$ Centrum voor Ontwikkelingsstoornissen, Faculteit Geneeskunde, Katholieke Universiteit, Leuven, Belgium. discussed. It is concluded that if the translation process is not adequately addressed, researchers may be guilty of simply imposing notions of health and quality of life across cultures. Consequently, the results will not provide meaningful insights into the cultures under study.
\end{abstract}

\section{KEYWORDS: TRANSLATION, SHONA, QUALITY OF LIFE, CULTURE}

\section{INTRODUCTION}

With the growing international collaboration in clinical research, there has been an increasing need for international instruments to assess outcomes (Bullinger et al 1998). Health related quality of life (HRQoL) measures are important outcome measures that are often used in international studies either to evaluate change over time or to discriminate between two groups at a specific point in time (Beaton et al 1996). The majority of established measures stem from Anglo-American literature (Bullinger et al 1998) and in Southern Africa, research is often undertaken which requires the use of these instruments. However, health status measures developed in one country cannot simply be translated for use in a different cultural context (Wagner et al 1998). The translation process should ensure that, the target version is not only semantically equivalent to the source version but also conceptually equivalent. In other words, the translation should not only reflect the correct linguistic interpretation of the source but, if the instrument is to be useful, it should reflect the domains that the target culture regards important (Herdman 1998).
One of the most commonly used measures of HRQoL is the European Quality of Life - 5 dimensions or EQ-5D. It is a generic single index measure, validated in several European countries which has been used to measure health for both clinical and economic appraisal (Bakker and van der Linden 1995). The EQ-5D is a deceptively simple instrument which includes a description of the respondents own health with regard to five domains of function; rating of own health by means of a visual analogue scale, and background information about the respondent (Brooks and EuroQol 1996) (See Table I). The EuroQol Group has developed a strict protocol to guide those who wish to translate the instrument from English into other languages (EuroQol). At present, there are many European language versions of the EQ-5D and a Japanese version. This report describes the first attempt to produce an officially approved version in the language of an African country.

The aim of this paper is to document the difficulties encountered in translating the EQ-5D into Shona, the language of the majority of the citizens of Zimbabwe. It is likely that similar problems are encountered in translating
Western developed instruments into the local Southern African languages and it is hoped that our experience will assist others in the development of locally valid translated instruments.

\section{BACKGROUND INFORMATION}

Zimbabwe is a country of approximately 12 million people. There are two main language groups, Shona and Ndebele. Shona speakers comprise the majority language group. English is the official language. Since Independence in 1980 , there has been free compulsory primary education (seven years of schooling) and the adult literacy rate is high, $91 \%$ (compared to $84 \%$ in South Africa) (Tandon 2000). Both Standard Shona and Ndebele are taught at schools and examined at both ' $O$ ' and ' $A$ ' level.

CORRESPONDENCE:

Jelsma J

Department of Rehabilitation,

University of Zimbabwe,

Box A178, Avondale,

Harare, Zimbabwe.

Tel: 263-4-791631.

Fax: 263-4-724912.

Email: jjelsma@healthnet.zw 
However, standard Shona is academic and elitist and no one in fact speaks it. There are several dialects, including Manica, Zezuru and Kalangu.

Prior to this translation, work had been done to establish the reliability of the EQ-5D in a high-density area in Harare (Chimera and Jelsma 1999). A translation of the EQ-5D had been carried out but the translation protocol had not been adequately followed to justify recognition as an official translation by the EuroQoL Group. As the EQ-5D was to be used as a measurement instrument in ongoing research on HRQoL, it was decided to redo the translation according to the protocol prescribed by the EuroQol Group.

\section{METHOD}

The protocol was followed as closely as possible. This entailed

i) Forward translation carried out independently by two native Shona speakers and a meeting to gain consensus on the first draft.

ii) Backward translation by two translators working independently and a meeting to gain consensus.

iii) Testing of the instrument on a lay panel of 18 participants.

At each stage of the process, reports of the meetings were sent to the EuroQol Group and comments and suggestions were made and implemented before progressing to the next phase.

The two forward translators were lecturers in the Department of English, one of whom spoke the Zezuru dialect and the other the Ndau dialect. It was agreed that the translation would be produced in standard Shona. Standard Shona is the meeting point of all dialects, equivalent to BBC English. It is the local lingua franca and suppresses the variance of different dialects. Similar to all standardised languages the difference between the use of different dialect speakers when speaking standard Shona lies not in expressions or choice of words but more in pronunciation: phonology and inflections, e.g. American English and British English.

The two forward translators worked independently of each other to produce a draft version. Translator 1 produced a more direct, literal translation. Translator 2 used more colloquial and natural oral discourse. He translated the content into the idiom and conceptual framework of the respondents to a greater extent. The problem with this translation was that back-translation into English might have produce unrecognisable results. These versions were extensively discussed and a consensus version agreed upon.

The Translation Protocol requires that the back translators be native English speakers and the EuroQol Group suggested that they did not necessarily have to be fluent in Shona but should have a reasonable command of Shona. However, we argued that we were not just addressing language issues but trying to interface completely different cultures and worldviews. Consequently, we felt that if we asked a native English speaker who would likely represent the Western Judeo-Christian point of view on illness and disability, we would lose out on the specific cultural nuances that are present in the choice of almost all the words. The translation would end up being a technically correct, but essentially foreign instrument.

The other argument that we put forward for the services of native Shona speakers as back translators was that English is the official language of Zimbabwe and that the back translators had not only completed British set and marked examinations but had also completed honours degrees in English. In contrast, in European and other countries, national languages are all spoken officially and at school. Any English speaking person living in these European countries would be forced to learn to speak these national languages. Similarly the nationals of these countries would not be constantly exposed to English.

The EuroQol Group accepted these arguments and the back translators appointed were then native Shona speakers, both of whom were lecturers in the English Language at the Open University of Zimbabwe. Once again, one translator was asked to produce a literal, direct translation and the other a more idiomatic translation.

The process took many hours of discussion. At each stage of the process, feedback was received from the EuroQol Translation Group and suggested modifications were implemented

\section{RESULTS AND DISCUSSION OF THE PROCESS}

The results of the back translation are presented in Table I.

\section{Conceptual aspects:}

It was apparent from the outset of the translation process that the concepts described by the EQ-5D do not sit comfortably in the Shona worldview. For example, the English concepts of health sound inappropriate when translated because some health states are seen to have a spiritual rather than a physical origin. Mararike (1999) states that illnesses may be normal (i.e. an illness with an impersonal cause) or abnormal (an illness with interpersonal causes.) Abnormal diseases can only be treated by traditional healers because modern medicine cannot address the ultimate cause of the illness, namely witches or the various types of spirits. (Mararike, 1999). Attitudes towards these health states are therefore understood and interpreted differently in Shona culture and experience. The traditional Shona way of life is largely spiritual and humanistic, within the group and less materialistic and individualistic than, perhaps, that of western capitalist societies (Mararike 1999). People define themselves in terms of the group and their health or illness is actualised within that context. The contrast to Western individualism and emphasis on self-determinism implies that independence in functioning is not weighted as heavily as it would be in Western cultural contexts and concepts related to personal care and usual activities are understood differently. In the next stages of validation of the instrument it might be necessary to establish the relative item weights (measurement equivalence) as these can be expected to reflect the comparative importance given to different items in different cultures (Herdman 1998).

\section{Semantic Equivalence:}

Several problems were encountered with achieving semantic equivalence, which is concerned with the transfer 
Table I: Results of the back translation

\begin{tabular}{l|l|l}
\hline ORIGINAL & FIRST TRANSLATOR & SECOND TRANSLATOR \\
& (LITERAL) & (ENGLISH IDIOM) \\
EQ - 5D Health Questionnaire & EQ - 5D & EQ -5D \\
(Shona version) & Health questionnaire & Questionnaire on Health
\end{tabular}

By placing a tick (thus) in one box in each group below, please indicate which statements best describe your own health state today.

\section{Mobility}

I have no problems in walking about.

I have some problems in walking about.

I am confined to bed.

\section{Selfcare}

I have no problems with self-care.

I have some problems in washing and dressing.

I am unable to bath or dress myself.

Usual activities (e.g. work, study, housework, family or leisure activities)

I have no problems performing my usual activities

I have some problems with performing my usual activities. I am unable to perform my usual activities.

\section{Pain/discomfort}

I have no pain or discomfort. I have moderate pain or discomfort. I have extreme pain or discomfort.

\section{Depression/anxiety}

I am not anxious or depressed.

I am moderately anxious or depressed.

I am extremely anxious or depressed.

In each set of the following responses insert a tick in th box at the end like this $[\checkmark$ ] against one answer only which you think is the one that explains your health today.

\section{Being able to walk/move}

I have no difficulty in walking/ moving.

Walking/moving is difficult for me.

I cannot even walk/move.

\section{Keeping/taking care of myself I am able to keep myself.}

I am not really able to wash myself or dress myself.

I am not able to wash or dress myself.

\section{Everyday tasks (For example:} working, reading/studying, doing work in the house or around the house, relaxing or spending leisure time with the family)

I have no problems doing the tasks I normally do every time.

I have problems doing tasks I normally do every day. I can no longer do the tasks I used to do every day.

\section{Pain/being unsettled}

I am not feeling pain.

I feel pain a little myself.

I feel pain very much.

Having trouble with the brain/thinking/being depressed I have nothing that troubles the brain.

I have many things that trouble me.

I am being troubled very much.
Choose one response, from each of the following sets, which most accurately describes the state of your health today. Place a tick in the box $[\checkmark]$ against your chosen response.

\section{Movement}

I feel no pain when I walk/move.

I feel pain when I walk.

I am unable to walk.

\section{Personal Hygiene}

I can look after my personal hygiene without any problem. I have difficulties in bathing or dressing.

I am unable to wash or dress myself.

\section{Performance of day to day tasks} (for example, work, studying, domestic work, entertaining family) I have no problems in doing my day to day duties.

I have problems in doing my day to day duties.

I am unable to do my day to day duties.

\section{Physical pain/discomfort} I feel no pain or discomfort. I feel moderate pain or discomfort. I have severe pain/ discomfort.

\section{Mental problems and anxieties}

I have no mental problems or anxieties.

I suffer from moderate stress/anxiety.

I suffer from severe stress/anxiety. 
Table I: Results of the back translation (continued)

\section{ORIGINAL \\ EQ - 5D Health Questionnaire (Shona version) \\ Compared with my general health over the last 12 months my present state of health today is: Better, Much the same, worse.}

Instructions for VAS

Your own health state today.

Best imaginable health state.

Worst imaginable health state

Because all replies are anonymous, it will help us to understand people's answers better if we have a little background data from everyone, as covered in the following questions:

Have you experienced serious illness? Yes [ ] No [ ] In yourself?

\section{FIRST TRANSLATOR (LTTERAL) \\ $E Q$ - 5D \\ Health questionnaire}

When I am comparing my health in the last twelve months and today, I can say myself today: It is now better. It is the same. It is worse.

So that we can help people to see how good or bad their health is today, we have given you a scale to measure (it looks like a thermometer used to measure body temperaturel, it has numbers starting from 0 up to 100.0 shows the poor health of someone who is very ill. 100 shows the very good health of someone who is not ill.

We ask you to point at the number on the scale that you think is the one that shows exactly where your health is today. Do this by drawing a line starting from the box that is below to your right, where the scale is, until you end where exactly the number you have chosen which you think is the one that shows where your health is today.

Your health today

Very good health

Very bad health

Because your answers will not have your names, we kindly ask you to tell us something about how you live your life from what follows. Insert a check in the box like this $[V]$ on each answer that you will have chosen

Have you ever seen someone very, very ill? Yes [ ] No [ ]

Are you the one yourself?

\section{SECOND TRANSLATOR (ENGLISH IDIOM) \\ EQ -5D \\ Questionnaire on Health}

When I look back at my health during the last twelve months, I would say that at present: I feel better than I have felt during the last twelve months. My state of health has not changed throughout the period. I feel worse that I have felt in the last twelve months.

To help respondents to accurately estimate their state of health, we have provided a scale (similar to a thermometer measuring body temperature) which ranges from 0 to 100.0 represents a very poor state of health, while 100 represents the height of good health. Mark on this scale the level at which you estimate your health to be. Draw a line from the box labelled "Your state of health today" to the number on the scale that most accurately reflects your state of health.

The state of your health at this moment

Very good health

Very bad health

Since this questionnaire will not carry your names, we ask you to provide us with additional information about your health which you may regard as confidential. Place a tick in the box against the most suitable response Have you ever seen a very ill person? Yes [ ] No [ ]. Was this person yourself? 
Table I: Results of the back translation (continued)

\begin{tabular}{|l|l|l|}
\hline ORIGINAL & FIRST TRANSLATOR & SECOND TRANSLATOR \\
& (LITERAL) & (ENGLISH IDIOM) \\
EQ $-5 D$ Health Questionnaire & EQ $-5 D$ & EQ -5D \\
(Shona version) & Health questionnaire & Questionnaire on Health
\end{tabular}

In your family.

Is it another member of your family?

In caring for others.

What is your age in years?

Are you Male Female?

Are you;

A current smoker

An ex-smoker

Never smoked

Do you now or did you ever,

work in health or social services?

If so, in what capacity?

Which of the following best

describes your main activity?

In employment or self-employment

Retired

Housework

Student

Seeking work

Other (please specify)

Did your education continue after the minimum school leaving age?

Do you have a degree or professional equivalent? If you know your postcode would you please write it here?

Is it someone you saw in your work for health and helping people that you were doing?

How many years do you have?

Are you a man or a woman?

Do you smoke?

Did you use to smoke?

Have you never smoked?

Do you work now, or have you ever worked as a health worker or someone who helps people? If that is true, what was your position?

Of the following, tell us what you can say is exactly something that you are doing:

Job that you do yourself or that you are employed to do

On pension

Domestic work

School child

Looking for work/employment

Any other that we have left out (tell us what it is)

Have you done further studies since you left/finished school?

Do you have a degree or any other course similar to a degree?

If you know your address, please write it here.

From the following indicate your main/major occupation:

Self employed or formally employed

Pensioner

Domestic Work

Student

Seeking employment

Any other occupation (not included above): Specify.

Did you further your education after leaving school?

Do you have a degree or it's equivalent?

If you know it, please indicate your address here.

of meaning across languages and with achieving a similar effect on respondents in different languages (Herdman 1998). Shona does not separate an abstract concept from the context in which it is experienced. E.g. The concept "pain" is not separate from the person who is experiencing the pain. Abstract concepts require either a subject or an object. Similarly causation is part of Shona idiom and it is difficult to simply say, "I have some problems with walking" without identifying the something which is giving me problems with walking. This is evidenced by the inclusion of "pain" in Translator 2's version ("I feel pain when I. walk"). The panel concluded that pain was not inherent in the words used and that the final condition (confined to bed) changes the implication from pain causing the problems to unspecified problems. Similarly it is difficult to say "Confined to bed" without stating the agent that is responsible. 


\section{Quantitication of degree of problem:}

The quantification of degree of problem was also difficult, a problem also experienced in the translation of SF-36 from American English into European languages (Wagner et al 1998) and examined further by Keller et al (1998). For example, a literal translation of "I have some problems with walking" is possible but the expression is unnatural in Shona. One is either mobile or immobile. The Shona would say, "I can walk a little" not "I have some problems with walking". The latter would imply some other kinds of problems with mobility, such as not having a car. Eventually the phrase was translated as "I can walk but I have some problems with doing so" or "Walking gives me problems" which implies ability to walk but with difficulty.

The innate modesty of the language also makes definitive statements unacceptable in Shona idiom. It implies arrogance to say, "I have no problems..." For example, the words used to translate "I have no problems with self-care" come across as arrogant, "I don't need anyone to help me". However, a slightly apologetic tone is introduced by the insertion of "zvangu" - "myself" which softens the statement and implies more modestly, "me, myself, I have no problems".

Similarly people would hesitate to say that their health state approximates the "Best imaginable health state" or "Worst imaginable health state" and these words were replaced with "Very good health" and "Very bad health".

\section{Different domains: \\ Mobility:}

Problems with the translation of mobility concepts have been discussed above. The translating of "confined to bed" presented two problems. As mentioned above, Shona requires an agent - who or what has confined the person to bed? Secondly, not everyone sleeps in a bed. In the end an idiomatic Shona expression was used which implies being unable to move out of bed. Literally the expression means "I cannot even walk at all" but it implies other problems apart from walking about and can be used metaphorically for a day in bed with an acute illness as well as a more permanent health state. It encompasses the idea of both short-term illness (e.g. flu) and long term paralysis. A more direct translation of the English "Confined to bed" implies that the person is moribund and about to die.

\section{Self care:}

Possible options included "kuzvibatsira" (to help oneself) or "kuzviitira" (to carry out tasks for oneself) or "kuzvishandira" (to work for oneself) or "kuzvionera" (to look after oneself). "Kuzvibatsira" was chosen as being closest to the original in implying some disability when difficulty is encountered with the tasks. This was literally back translated as "I am able to keep myself". The potential confusion between the wider sense of taking care of oneself (i.e. fending for oneself) is limited to personal care by the inclusion of "washing and dressing" in the next question.

\section{Pain/discomfort}

Discomfort was difficult to translate and expressions such as "I cannot even stay in one place" were considered. Another issue is that in Shona, discomfort is not a sensation separate from pain. Once the idea of discomfort has been included, the word pain in the rest of the questions "kurwadziwa" includes the concept of discomfort.

\section{Depression/ anxiety}

Of all the EuroQol concepts, the idea of depression and anxiety are most difficult to capture in Shona. There is no specific word for depression, it is usually implied from symptoms rather than self report. Anxiety and depression are the meanings of the same word, "kuremerwa". Depression is seen to be caused by some life experience and is not described as a psychological state in isolation. While both anxiety and depression may cause sadness, they do not necessarily incorporate sadness in themselves. Anxiety and depression become premonitions where the causes are not palpable. Anxiety and depression are not, therefor health states in Shona. They are understood as occasional psychological (social/alienation) or spiritual (religious) states. In addition, severe anxiety is seen to border on a psychiatric state known as "mhopu".
After extended discussion, the words literally translated as "I have nothing that troubles the brain" or idiomatically translated into the English "I have no mental problems or anxieties" were accepted.

\section{Courtesy:}

The question relating to the age of the respondent also posed a problem as in normal social discourse it would be extremely discourteous to ask such a question of an adult. The more usual framing of this question would be "In what age bracket do you belong?" which is in fact a socialisation question. The answers would then be in the twenties or thirties, adult teenager or even married that would imply adult.

\section{Contextud issues:}

Although not dealt with directly in the translation process, issues such as the setting of the interview, as well as the gender and ages of the interviewer and respondent respectively can have a major impact on the outcome of the questionnaire and these issues need careful consideration.

\section{CONCLUSION}

Although it is likely that the Shona respondents will identify it as a foreign instrument, Shona is able to capture the EQ-5D concepts. The respondents will be able to recognise the concepts and respond appropriately, while maintaining the awareness that the questions do not relate directly to "their world". The translation attempted to make the sense, meaning, spirit and practice, which the concepts and expressions in English signify, understood by target language speakers in Shona, as clearly and naturally as possible. However, it is conceded that the target speakers might see, experience and practice them quite differently. Accurate translation of the source instrument is only the first stage of the process of developing a comparable version in the target language. The Shona EQ-5D still needs to be validated in terms of psychometric criteria of reliability, validity and responsiveness (Bullinger et al 1998).

The relative advantages of developing a homegrown instrument and of 
translating existing instruments would need to be examined in every research context. However a strong argument for the translation of standardised instruments was made at a regional workshop on research informed rehabilitation planning in which it was stated

"An issue of regional concern is that the lack of standardised methods and terminology make it virtually impossible to validate the different results and certainly makes it impossible to compare the results of the different regional studies." (Ferrinho and Cornielje 1998)

Our experience supports Herdman's contention that conceptual equivalence should not be assumed on the basis of measurement properties and correlations obtained using translated questionnaires, but also by evaluating the questionnaires themselves in the light of knowledge regarding the HRQoL concept in the target culture (Herdman 1998). We conclude that if an instrument is to be used in a culture, which differs widely from the source culture, extensive discussion needs to take place at every stage of the translation process. Both forward and back translators should be native speakers of the target language, particularly if English is the official language of the target population (as is the case in Southern Africa). The utilisation of translators from outside the medical field contributed to a more natural and idiomatic version of the instrument. In addition the extensive knowledge of the target culture that the translators enjoyed, resulted in a culturally more acceptable translation.

At the initiation of the project, the EQ-5D was chosen as a suitable instrument for measurement of HRQoL because it had been validated over a wide range of conditions and it is simple and easy to understand. In the light of the difficulties involved in translating what appears to be a short and relatively straightforward instrument, we would advise researchers to think very carefully about choosing instruments that are longer and more complex. The more complex the instrument is, the more difficult it will be to develop a local translation that is conceptually and semantically equivalent.

In conclusion, time spent in following a stringent translation protocol, such as developed by the EuroQol Group, is time well spent. In Southern Africa, there is an exciting interface between cultures, languages and worldviews. If relevant research is to be done in the sub-continent, a meeting place between English and the local languages, between AngloAmerican culture and traditional ways of living and between the Western Medical model of health and traditional cultural beliefs needs to be established and nurtured. The development of accurate and culturally sensitive instruments, either through careful translation of existing instruments or through the development of locally relevant measures, can be an important embarkation point. If the translation process is not taken seriously, we may be guilty of simply imposing notions of health and quality of life across cultures (Herdman 1998) and our results will not provide meaningful insights into the cultures that we are studying.

\section{ACKNOWLEDGEMENTS:}

The comments and suggestions by Michael Herdman at each stage of the translation process are gratefully acknowledged. Funding for the project was made available by the Zimbabwe National Burden of Disease Steering Committee funded by the DANIDA, Danish Development Agency and DFID, the British Development Agency.

\section{REFERENCES}

Bakker C, van der Linden S 1995 Health related utility measurement: An introduction. Journal of Rheumatology 22: 1197-1199

Beaton DE, Bombardier C, Hogg-Iohnson SA 1996 Measuring health in injured workers: A cross-sectional comparison of five generic health status instruments in workers with musculo-skeletal injuries. American Journal of Industrial Medicine 29: 618-631

Brooks R, EuroQol G 1996 EuroQol: the current state of play. Health Policy 37: 53-72

Bullinger M, Alonso J, Apolone G, Leplege A, Sullivan M 1998 Translating health status questionnaire and evaluating their quality: The IQoLA project approach. Journal of Clinical Epidemiology 51: 913-923

Chimera J, Jelsma J 1999 The reliability of the EuroQol in a high-density suburb in Harare, Zimbabwe. BSc. (Hons.) thesis. University of Zimbabwe
EuroQol Undated. Translation guidelines for the EuroQol EQ-5D, EuroQoL Group, Rotterdam

Ferrinho P, Cornielje H 1998 Towards consensus on research-based disablement policies for Southern Africa. Proceedings of the Workshop "Towards Research Informed Rehabilitation Planning in Southern Africa", Harare, Zimbabwe. Bulawayo, SAFOD 117-123

Herdman M 1998 A model of equivalence in the cultural adaptation of HRQoL instruments: the universalist approach. Quality of Life Research 4: 323-335

Keller S, Ware JE, Gandek B, Aaronson N, Alonso J 1998 Testing the equivalence of translations of widely used response choice labels: Results from the IQoLA Project. Journal of Clinical Epidemiology 54: 933-944

Mararike CG 1999 Survival strategies in rural Zimbabwe. Harare: Mond Books ,
Tandon Y. 2000. Human Development Report 1999 - Zimbabwe, United Nations Development Programme, Poverty Reduction Forum, Institute of Development Studies, University of Zimbabwe, Harare

Wagner AK, Gandek B, Aaronson N, Acquadro C, Alonso J 1998 Cross-cultural comparisons of the content of SF-36 translations across 10 countries: Results from the IQoLA project. Journal of Clinical Epidemiology 51: 925-32 